\title{
Leitthema
}

Bundesgesundheitsbl 2013 · 56:1260-1269

DOI 10.1007/s00103-013-1801-7

Online publiziert: 30. August 2013

(c) Springer-Verlag Berlin Heidelberg 2013

O. Wichmann ${ }^{1}$ - B. Ultsch ${ }^{1,2}$

${ }^{1}$ Fachgebiet Impfprävention, Abteilung für Infektionsepidemiologie, Robert Koch-Institut, Berlin

${ }^{2}$ Charité Universitätsmedizin Berlin

\section{Effektivität, Populationseffekte und Gesundheitsökonomie der Impfungen gegen Masern und Röteln}

Masern und Röteln sind hochkontagiöse Viruserkrankungen, die von Mensch zu Mensch übertragen werden. Während mit Masern Komplikationen wie Pneumonie (1-6\% der Fälle), Enzephalitis (1 pro 1000 bis 2000 Masernfälle) oder Tod ( 1 bis 3 pro 1000 Masernfälle) assoziiert sind, ist bei der üblicherweise mild verlaufenden Rötelnvirusinfektion die Embryofetopathie nach Infektion in der Schwangerschaft gefürchtet [1]. Seit Anfang der 1960er-Jahre sind zum Schutz vor diesen beiden Erregern lebend-attenuierte Impfstoffe zugelassen, die seit mehreren Jahrzehnten zum Standard der meisten nationalen Impfprogramme gehören. Der Erfolg der Impfmaßnahmen ist enorm und mündete in Nord- und Südamerika in die Elimination beider Erkrankungen [2]. Aufgrund unzureichender Impfquoten oder Impfprogramme in anderen Regionen starben jedoch $2010 \mathrm{im}$ mer noch geschätzte 139.300 Kinder weltweit an Masern, und 2008 wurden vermutlich mehr als 80.000 Kinder mit einem kongenitalen Rötelnsyndrom geboren [3, 4]. Die Epidemiologie der Masern und Röteln in Deutschland ist in einem gesonderten Beitrag in diesem Schwerpunktheft beschrieben.

Aufgrund der weltweit beträchtlichen Krankheitslast, aber auch aufgrund der biologischen Machbarkeit ist die Elimination beider Erkrankungen in mindestens 5 Regionen der Weltgesundheitsorganisation (WHO) bis 2020 vorgesehen [5]. Im vorliegenden Beitrag soll die Evidenz für die Effektivität der Impfung gegen Röteln und Masern dargestellt werden. Ein aus- führliches Cochrane-Review zu diesem Thema wurde 2012 publiziert, sodass hier auf diese Ergebnisse zurückgegriffen und auf die Durchführung eines eigenen Reviews verzichtet wurde [6]. Da das Cochrane-Review jedoch nur die Effektivität der Masern-Mumps-Röteln (MMR)Kombinationsimpfstoffe berücksichtigt, wurden ergänzend Ergebnisse aus einem systematischen Review zu Effektivität der Masernimpfstoffe aus dem Jahr 2011 und aus dem WHO-Positionspaper zu den Rötelnimpfstoffen von 2011 berücksichtigt $[4,7]$. Die Suchstrategien sowie Einund Ausschlusskriterien sind in den jeweiligen Publikationen zu finden.

Zusätzlich sollen in diesem Artikel die epidemiologischen Effekte der beiden Impfungen auf Bevölkerungsebene nach ihrer Integration in Impfprogramme und ihre gesundheitsökonomischen Konsequenzen beschrieben werden. Der Effekt auf die Krankheitslast auf Bevölkerungsebene wird anhand ausgesuchter Beispiele beschrieben: i) in Ländern des amerikanischen Kontinent (als Beispiel für die bislang einzige erfolgreiche regionale Elimination), ii) in Finnland (als Beispiel für ein europäisches Land, in dem die Elimination bereits gelungen ist) und iii) in Deutschland (auch um hier die Unterschiede in der Impfstrategie im Vergleich zu den vorher genannten Bespielen herauszuheben). Zur Beschreibung der gesundheitsökonomischen Konsequenzen wurden am 17.01.2013 2 systematische Literaturrecherchen in der Datenbank Medline durchgeführt, um gesund- heitsökonomische Analysen zur Masernund Rötelnimpfung zu identifizieren. Die verwendete Suchstrategie basierte auf der Verwendung von „Medical Subject Headings" (Mesh-Begriffen). Mit der Strategie

(„Measles"[Mesh] OR „MeaslesMumps-Rubella Vaccine"[Mesh] OR „Measles Vaccine“[Mesh] OR „Measles virus"[Mesh]) AND („Costs and Cost Analysis"[Mesh] OR „Economics"[Mesh] $O R$,economics“[Subheading] OR „CostBenefit Analysis"[Mesh] OR "Cost of Illness"[Mesh] OR „Cost Savings"[Mesh] OR ,Health Care Costs"[Mesh] OR „Direct Service Costs“[Mesh] OR „Hospital Costs"[Mesh] OR „Employer Health Costs"[Mesh] OR „Drug Costs"[Mesh]) AND (,humans"[MeSH Terms] AND (English [lang] OR German[lang])) wurden 339 Publikationen mit Masernbezug identifiziert. Nach Analyse der Titel bzw. Abstracts wurden 331 Studien ausgeschlossen, da sie nicht die Einschlusskriterien "gesundheitsökonomische Analyse der Masern- bzw. MMR-Kombinationsimpfung in einem Industrieland außerhalb einer Ausbruchsphase" erfüllten. Die 8 eingeschlossenen Studien $[8,9,10,11,12$, $13,14,15]$ wurden um eine weitere Studie [16], die mittels Handsuche identifiziert wurde, ergänzt. Mit der Strategie

(„Rubella“[Mesh] OR „MeaslesMumps-Rubella Vaccine“[Mesh] OR „Rubella Vaccine"[Mesh] OR „Rubella virus"[Mesh]) AND („Costs and Cost Analysis"[Mesh] OR „Economics"[Mesh] $O R$,economics"[Subheading] OR „CostBenefit Analysis"[Mesh] OR „Cost of Ill- 
Tab. 1 Definitionen und übliche methodische Ansätze zur Berechnung der Impfstoffwirksamkeit, -effektivität und -kosteneffektivität

\begin{tabular}{|c|c|c|c|}
\hline Größe & $\begin{array}{l}\text { Ansatz/Stu- } \\
\text { diendesign }\end{array}$ & Berechnungsformel & Definition/Anmerkung (basierend auf $[21,26,34]$ ) \\
\hline $\begin{array}{l}\text { Impfstoff- } \\
\text { wirksamkeit } \\
\text { (engl.,,vaccine } \\
\text { efficacy“) }\end{array}$ & $\begin{array}{l}\text { Rando- } \\
\text { misierte, } \\
\text { kontrollierte } \\
\text { Studie (RCT) }\end{array}$ & Wirksamkeit $($ in $\%)=\frac{(I R U-I R G)}{I R U} \cdot 100=(1-\mathrm{RR}) \cdot 100$ & $\begin{array}{l}\text { Definition: Direkter Effekt der Impfung unter kontrollierten } \\
\text { (optimalen Studien-)Bedingungen } \\
\text { Prozentuale Reduktion der Inzidenzrate (IR) der Zielerkran- } \\
\text { kung unter geimpften (IRG) im Vergleich zu ungeimpften } \\
\text { (IRU) Individuen. Die Wirksamkeit kann auch direkt aus } \\
\text { dem relativen Risiko (RR) der Geimpften in Bezug auf die } \\
\text { Zielerkrankung im Vergleich zu Ungeimpften berechnet } \\
\text { werden }\end{array}$ \\
\hline \multirow[t]{3}{*}{$\begin{array}{l}\text { Impfstoff- } \\
\text { effektivität } \\
\text { (engl.,,vaccine } \\
\text { effectiveness") }\end{array}$} & $\begin{array}{l}\text { Retrospekti- } \\
\text { ve Kohorten- } \\
\text { studie }\end{array}$ & Effektivität (in \%) $=\frac{(I R U-I R G)}{I R U} \cdot 100=(1-\mathrm{RR}) \cdot 100$ & $\begin{array}{l}\text { Definition: Direkte und indirekte Effekte der Impfung unter } \\
\text { Feldbedingungen (z. B. im Rahmen eines Impfprogramms) } \\
\text { In der Kohortenstudie entspricht die Effektivität der prozen- } \\
\text { tualen Reduktion der Inzidenzrate (IR) der Zielerkrankung } \\
\text { unter geimpften (IRG) im Vergleich zu ungeimpften (IRU) } \\
\text { Individuen }\end{array}$ \\
\hline & $\begin{array}{l}\text { Fall-Kontroll- } \\
\text { Studie }\end{array}$ & Effektivität (in \%) $=(1-\mathrm{OR}) \cdot 100$ & $\begin{array}{l}\text { In der Fall-Kontroll-Studie geht der Impfstatus (geimpft } \\
\text { bzw. ungeimpft) von Erkrankungsfällen und Nicht-Erkran- } \\
\text { kungsfällen (Kontrollen) als Odds Ratio (OR) in die Analyse } \\
\text { ein }\end{array}$ \\
\hline & $\begin{array}{l}\text { Screening- } \\
\text { methode }\end{array}$ & Effektivität (in \%) $=\frac{(P B G-P F G)}{P B G \cdot(1-P B G)} \cdot 100$ & $\begin{array}{l}\text { Die Screeningmethode erlaubt eine grobe Abschätzung der } \\
\text { Impfeffektivität, für die der Anteil an Geimpften in der Ziel- } \\
\text { population (Prozentanteil Bevölkerung geimpft, PBG) und } \\
\text { unter den Erkrankungsfällen (PFG) gekannt sein muss }\end{array}$ \\
\hline $\begin{array}{l}\text { Kosten- } \\
\text { effektivität } \\
\text { (engl.,,,cost ef- } \\
\text { fectiveness") }\end{array}$ & $\begin{array}{l}\text { Inkrementel- } \\
\text { les Kosten- } \\
\text { Effektivitäts- } \\
\text { Verhältnis }\end{array}$ & 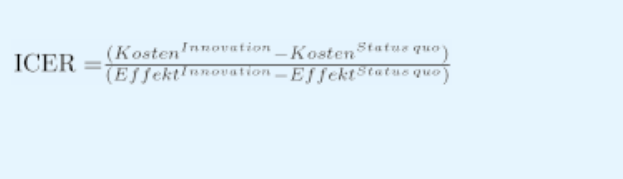 & $\begin{array}{l}\text { Zur Berechnung und Darstellung der Kosten-Effektivi- } \\
\text { täts- bzw. Kosten-Nutzwert-Relationen zwischen der } \\
\text { innovativen Impfung und dem Status quo gelangt man } \\
\text { über die inkrementelle Kosten-Effektivitäts-Relation (engl.: } \\
\text { incremental cost-effectiveness ratio, ICER) }\end{array}$ \\
\hline
\end{tabular}

ness“[Mesh] OR „Cost Savings“[Mesh] OR „Health Care Costs"[Mesh] OR „Direct Service Costs“[Mesh] OR „Hospital Costs“[Mesh] OR „Employer Health Costs“[Mesh] OR „Drug Costs“[Mesh]) AND ("humans"[MeSH Terms] AND (English[lang] OR German[lang])) wurden 188 Publikationen mit Rötelnbezug identifiziert. Von diesen Studien wurden nach Bewertung der Titel und Abstracts 4 Studien $[17,18,19,20]$ in die Analyse eingeschlossen. Diese entsprachen der Absicht der Recherche, Studien zum Thema „gesundheitsökonomische Analyse der Röteln- bzw. MMR-Kombinationsimpfung in einem Industrieland außerhalb einer Ausbruchsphase" zu identifizieren.

\section{Effekte von Impfungen sowie Methoden ihres Nachweises}

Impfungen können auf individueller, aber auch auf Bevölkerungsebene sowohl erwünschte als auch - in seltenen Fällen unerwünschte Effekte (z. B. unerwünschte Arzneimittelwirkungen, aber auch Serotypen-Replacement oder Verschiebung der Krankheitslast) verursachen. Üblicherweise hat eine Impfung den direkten Effekt, das geimpfte Individuum vor der Zielerkrankung zu schützen oder ihren klinischen Verlauf abzuschwächen. Gelegentlich bildet sich jedoch aufgrund eines primären Impfversagens kein Schutz aus.

Nach breiter Anwendung einer Impfung in der Bevölkerung kann es bei vielen impfpräventablen Erkrankungen zu einer Reduktion der Transmission und so zu indirekten Effekten (auch Herdeneffekte genannt) kommen. Von diesen indirekten Effekten können dann nicht geimpfte Individuen, aber auch geimpfte Personen, die keinen direkten Schutz ausgebildet haben, profitieren. Der Gesamteffekt eines Impfprogramms setzt sich somit zusammen aus einem indirekten Effekt auf ungeimpfte Individuen und aus direkten und indirekten Effekten auf geimpfte Individuen [21].

\section{Wirksamkeit und Effektivität von Impfungen}

In der englischsprachigen Literatur unterscheidet man den Terminus ,effica- cy“ (im Folgenden Wirksamkeit genannt) vom Terminus "effectiveness“ (im Folgenden Effektivität genannt).

Üblicherweise wird von der Wirksamkeit im Rahmen von klinischen Studien gesprochen, bei denen i) der Impfstoff unter optimalen Bedingungen appliziert wird und ii) aufgrund einer limitierten Anzahl an Studienteilnehmern nur direkte Impfeffekte zu erwarten sind [22]. Als Goldstandard-Methode zur Messung der Impfstoffwirksamkeit gilt die doppelblinde, randomisierte, placebokontrollierte Studie (englisch: randomized controlled trial; RCT). Der methodische Ansatz zur Berechnung der Wirksamkeit aus RCTs ist in $\bullet$ Tab. 1 dargestellt. Durch dieses Studiendesign wird das Risiko minimiert, dass der nachgewiesene Effekt einer systematischen Verzerrung (Bias) unterliegt. Daten aus RCTs werden üblicherweise für die Zulassung neuer Impfstoffe von den zuständigen Behörden gefordert. Dabei dienen RCTs sowohl dem Nachweis der Wirksamkeit als auch der Sicherheit des Impfstoffs. Die Wirksamkeit der Impfung kann in Bezug auf verschiedene kli- 
nische Endpunkte (z. B. Reduzierung von Erkrankungsfällen oder Hospitalisierungen) getestet werden, die in einer Kohorte von geimpften und ungeimpften Studienteilnehmern prospektiv über einen gewissen Zeitraum erfasst werden. $\mathrm{Zu}$ den Nachteilen von RCTs gehören ihre Komplexität, Dauer und Kostenintensität. Bei seltenen Zielerkrankungen muss die Studienpopulation entsprechend groß sein, um statistisch belastbare Ergebnisse zu erzielen. Falls eine Erkrankung äußerst selten auftritt (beispielsweise invasive Meningokokkenerkrankung), können auch Immunogenitätsstudien durchgeführt werden, sofern die Antikörpertiter positiv mit der klinischen Wirksamkeit korrelieren.

Der Terminus „Effektivität“ hingegen wird im Rahmen von epidemiologischen Beobachtungsstudien benutzt und spiegelt sowohl direkte als auch indirekte Effekte einer Impfung wider, die sich in einer Bevölkerung nach breiter Anwendung unter nicht immer optimalen Bedingungen (z. B. bei der Impfstofflagerung oder Verabreichung) ausbilden (- Tab. 1). Notwendig werden solche Studien unter anderem, um Phänomene zu untersuchen, die aufgrund der limitierten Probandenanzahl oder Studiendauer nicht in RCTs nachweisbar waren (z. B. nachlassender Impfschutz nach vielen Jahren), oder wenn es im Rahmen eines Impfprogramms zu einer unerwartet hohen Anzahl an Impfdurchbrüchen kommt.

Im Vergleich zu RCTs haben Beobachtungsstudien jedoch ein deutlich größeres Potenzial für systematische Verzerrungen, sodass bei ihrer Planung und Durchführung mehrere Faktoren (z. B. bei der Wahl der Falldefinition oder Kontrollgruppen) unbedingt berücksichtigt werden müssen [23, 24]. Zu den Studiendesigns, die typischerweise zur Erhebung der Impfeffektivität benutzt werden, zählen retrospektive Kohortenstudien und Fall-Kontroll-Studien. Retrospektive Kohortenstudien können insbesondere im Rahmen von institutionellen Ausbrüchen (z. B. an Schulen) oder unter Haushaltskontakten (Inzidenz von Sekundärfällen) durchgeführt und die Effektivität berechnet werden (• Tab. 1). Eine einfache und schnelle Schätzung der Effektivität einer Impfung nach breiter Anwendung in der

Bundesgesundheitsbl 2013·56:1260-1269 DOI 10.1007/s00103-013-1801-7

(c) Springer-Verlag Berlin Heidelberg 2013

\section{O. Wichmann · B. Ultsch}

\section{Effektivität, Populationseffekte und Gesundheitsökonomie der Impfungen gegen Masern und Röteln}

\section{Zusammenfassung}

Seit mehreren Jahrzehnten gehört die Impfung gegen Masern und Röteln zum Standard der meisten nationalen Impfprogramme. Im vorliegenden Übersichtsbeitrag sollen die Evidenz für die Effektivität der beiden Impfungen auf Basis veröffentlichter systematischer Reviews dargestellt sowie die epidemiologischen und gesundheitsökonomischen Gesamteffekte der Impfung auf Bevölkerungsebene beschrieben werden. Zahlreiche epidemiologische Beobachtungsstudien belegen die gute Effektivität (>90\%) beider Impfungen. Die Reduktion der weltweiten Maserntodesfälle sowie die dramatische Reduktion der Masern- und Rötelnkrankheitsinzidenz nach Einführung der Impfungen tragen zu einer sehr hohen Qualität der Evidenz bei. Die Länder des amerikanischen Kontinents konnten beweisen, dass durch sehr hohe Impfquoten in einem Routineimpfprogramm mit 2 Masern/Röteln-Impfdosen und mit zusätzlichen Impfaktivitäten zur Schließung von Immunitätslücken in höheren Altersgruppen eine Eliminierung beider Erkrankungen möglich ist. Eine gesundheitsökonomische Evaluation der Masern- bzw. Rötelnimpfung speziell für Deutschland gibt es nicht. Im Rahmen eines eigenen systematischen Reviews wurden jedoch 11 gesundheitsökonomische Studien aus anderen Industrieländern und eine für ein hypothetisches Industrieland identifiziert. In allen wurden sowohl der Masern- als auch der Rötelnimpfung stets ein kosteneffektives und teils sogar ein kostensparendes Potenzial zugerechnet, sodass dieses mit Einschränkungen auch für Deutschland anzunehmen ist. Zusammenfassend besteht ausreichend Evidenz für die hohe Effektivität der beiden Impfungen, mit denen die Masern- und Rötelneliminierung machbar ist, sofern eine adäquate Impfstrategie umgesetzt wird. In Deutschland sind dazu breit angelegte und koordinierte Maßnahmen zu Nachholimpfungen unter Kindern, Jugendlichen und jungen Erwachsenen speziell in den westlichen Bundesländern dringend notwendig.

\section{Schlüsselwörter}

Masern · Röteln · Impfung · Wirksamkeit · Kosteneffektivität

\section{Effectiveness, population-level effects, and heath economics of measles and rubella vaccination}

\section{Abstract}

Vaccination against measles and rubella has been included in national immunization programs worldwide for several decades. In this article, we present the evidence related to the effectiveness of measles and rubella vaccination based on published systematic reviews, and we describe the epidemiological and health economic effects of vaccination at a population level. Several observational studies demonstrate the high effectiveness (>90\%) of both measles and rubella vaccination. The global measles mortality reduction and the dramatic decrease in rubella and measles incidences after introduction of routine immunization contribute to the very high quality of evidence. The countries of the Americas have proved that it is feasible to eliminate measles and rubella by strengthening infant immunization through routine vaccination services and by conducting supplemental immunization activities in other childhood age groups so as to close immunity gaps. An economic evaluation of measles and rubella vaccination specifically for the healthcare system in Germany does not exist. However, we conducted a systematic review and identified 11 health-economic studies from other industrialized countries and one for a hypothetical industrialized country. Results indicate that vaccination against measles and rubella had either a cost-effective or even a cost-saving potential, which could be assumed with some limitations also for the German setting. In conclusion, there is compelling evidence that the available vaccines are very effective and that measles and rubella elimination is feasible if adequate vaccination strategies are implemented. In Germany, catch-up vaccination programs are urgently needed for children, adolescents, and young adults specifically in the western federal states.

\section{Keywords}

Measles · Rubella · Vaccination · Effectiveness . Cost-effectiveness 
Bevölkerung ist auch mittels der Screeningmethode möglich (• Tab. 1). Die Methode ist aber recht anfällig für Verzerrungen, insbesondere bei sehr niedrigen oder sehr hohen Impfquoten [22, 25]. Weitere Studiendesigns zur Abschätzung der Impfeffektivität sind möglich, sollen hier aber nicht weiter beschrieben werden $[23,26]$.

\section{Einfluss der Impfung \\ auf Bevölkerungsebene und in Ausbrüchen}

Das primäre Ziel von Impfprogrammen oder Impfempfehlungen ist die Inzidenzreduktion der Zielerkrankung in der Bevölkerung. Daher sollte jedes Impfprogramm bzw. jede Impfempfehlung durch ein Krankheitssurveillancesystem (z. B. mittels Meldepflicht oder in Sentinelsystemen) begleitet werden, um ihren Einfluss auf die Krankheitsinzidenz zu überwachen und evtl. Anpassungen der Impfstrategie zu ermöglichen. Über die die Krankheitssurveillance wird der Gesamteffekt einer Impfung auf Bevölkerungsebene abgebildet. Der Gesamteffekt wird durch die erreichten Impfquoten, die Verteilung der geimpften Individuen in der Bevölkerung und die Mischung von Bevölkerungsgruppen, aber auch durch die Effektivität und Schutzdauer der Impfung, die Bevölkerungsimmunität aufgrund natürlicher Infektion und durch die Erregercharakteristika wie die Basisreproduktionszahl $\left(\mathrm{R}_{0}\right)$ beeinflusst [21].

Die Reduktion der Krankheitsinzidenz kann bei Erreichen einer sehr hohen Bevölkerungsimmunität und Ausbildung von Herdeneffekten so ausgeprägt sein, dass - sofern der Mensch das einzige Erregerreservoir darstellt - eine endemische Transmission unterbrochen wird. Die Elimination einer Erkrankung in einer geografischen Region wird somit ermöglicht. Bei Masern bilden sich Herdeneffekte jedoch aufgrund der hohen Basisreproduktionszahl erst bei sehr hohen Impfquoten aus, sodass zur Elimination Impfquoten von $\geq 95 \%$ mit 2 Impfstoffdosen erforderlich sind [5].

Im Rahmen von Ausbrüchen impfpräventabler Erkrankungen stellen Impfaktivitäten neben nichtpharmakologischen Maßnahmen (z. B. Kontaktreduk- tion) eine Methode der Ausbruchskontrolle dar. Ihr Erfolg hängt maßgeblich von mehreren Faktoren ab: i) vom Grad der vorbestehenden Immunität in der Bevölkerung bzw. in der betroffenen Institution; ii) wie schnell der Impfstoff zur Verfügung steht und verabreicht werden kann; iii) wie schnell sich nach Impfung ein Schutz der geimpften Person ausbildet, und iv) von der Basisreproduktionszahl $\left(\mathrm{R}_{0}\right)$ des Erregers. Der Effekt von Impfmaßnahmen ist nur schwer quantifizierbar, da jeder Ausbruch anders ist und so nur schwer tatsächliche Szenarien mit und ohne Impfintervention verglichen werden können. Man kann jedoch mathematische Modellierungen durchführen, um ein tatsächliches Szenario mit einem hypothetischen Szenario zu vergleichen.

\section{Gesundheitsökonomie Evaluation von Impfungen}

Bei der gesundheitsökonomischen Evaluation von Impfstoffen werden mehrere Alternativen miteinander verglichen: in der Regel eine (innovative) Impfung mit dem Status quo (Standardimpfung oder Nichtimpfung). Da zum Zeitpunkt der Zulassung eines Impfstoffes in der Regel Unsicherheit über seine tatsächlichen Effekte auf Bevölkerungsebene (z. B. erwartete Reduktion von Krankheitsfällen, Hospitalisierungen und Todesfälle) und die Kosten bzw. vermiedenen Behandlungskosten durch die Impfung besteht $[27,28]$, ist speziell im Zusammenhang mit Impfungen eine Projektion in die Zukunft erforderlich. Daher werden für derartige Analysen mathematische Modellierungen durchgeführt, die derartige Prognoseunsicherheiten abbilden, indem sie in den Vorausberechnungen und Simulationen verschiedene Faktoren variieren und damit ihren Einfluss auf die Vorhersage testen [27, 29]. Zum anderen helfen diese Modellierungen, die effizienteste Impfstrategie (beispielsweise Beschränkung auf gewisse Alters- oder Risikogruppen) zu identifizieren und mit Alternativen zu vergleichen [30]. Speziell Phänomene wie Herdenimmunität, Serotypen-Replacement, Altersverschiebung der Krankheitslast und die Variation der epidemischen Zyklen, die gerade bei einer beinahe Ausrottung von impfpräventablen Krankheiten auftreten, können mit dynamisch mathematischen Modellen dargestellt und analysiert werden [27, 30, 31]. Das Phänomen der Clusterbildung kann mittels umfangreicher mathematischer Modellierungen ebenfalls zumindest theoretisch dargestellt werden. Die Clusterbildung basiert auf einer heterogenen Impfabdeckung in der Bevölkerung, die dazu führen kann, dass sich in einem Land mit generell hoher Impfquote einzelne geografische Regionen (z. B. Landkreise) mit niedrigerer Impfquote ausbilden. Krankheitsfälle können aus Ländern mit höherer Krankheitslast in diese Cluster von Empfänglichen importiert werden und somit zu geografisch beschränkten Ausbrüchen führen, was eine Elimination erschwert [32]. Mathematische Modellierungen können die epidemiologischen Auswirkungen (Dauer und Umfang eines Ausbruchs) innerhalb eines Clusters oder Landes mit heterogener Impfabdeckung berechnen [33]. Hierfür sind jedoch besonders Inputdaten wie (internationale) Reiseaktivitäten und Kontaktmuster innerhalb einer Bevölkerung nötig, um den Import und die potenzielle Ausbreitung von Erregern in einer empfänglichen (Teil-)Bevölkerung abbilden zu können $[33,34,35]$.

Als Inputdaten sind direkte medizinische Kosten zur Behandlung der Zielerkrankung, direkte nichtmedizinische Kosten, wie z. B. Transportkosten der Patienten, aber auch indirekte Kosten wie Arbeitsausfall aufgrund von Krankheit oder Arbeitsausfall aufgrund der Pflege erkrankter Angehöriger in den Modellrechnungen zu berücksichtigen $[34,36]$. Die Kosten der Impfung werden in Form direkter medizinischer Kosten berücksichtigt. Neben diesen ökonomischen Inputdaten bedarf es auch epidemiologischer Daten zur Krankheitsinzidenz, zur Lebenserwartung und Hintergrundmortalität sowie auch zur Impfeffektivität in der Zielpopulation. Je nach Zielrichtung der Modellierung kann auch die gesundheitsbezogene Lebensqualität von Gesunden und an der Zielerkrankung leidenden Individuen als Inputparameter eingeschlossen werden [37].

Als Output aus dem Vergleich der Impfsituation mit dem Status quo kön- 
Tab. 2 Übersicht der Ergebnisse zweier aktueller systematischer Reviews zur Effektivität der Masernimpfung

\begin{tabular}{|c|c|c|c|c|c|c|c|}
\hline Ort/Studienzeit & $\begin{array}{l}\text { Anzahl } \\
\text { Impfdosen }\end{array}$ & Alter bei Impfung & $\begin{array}{l}\text { Anzahl/Design einge- } \\
\text { schlossene Studien }\end{array}$ & $\begin{array}{l}\text { (Mediane) Impf- } \\
\text { effektivität }\end{array}$ & $\begin{array}{l}\text { 95\%-Konfi- } \\
\text { denzintervall }\end{array}$ & $\begin{array}{l}\text { Interquartile } \\
\text { Spanne }\end{array}$ & $\begin{array}{l}\text { Autor und } \\
\text { Jahr der Pub- } \\
\text { likation }\end{array}$ \\
\hline \multicolumn{8}{|c|}{ Im Cochrane Review eingeschlossene Studien zur Masernimpfeffektivität von MMR-Kombinationsimpfstoffen (Demicheli et al. 2012)a [6] } \\
\hline Italien 1996 & $\begin{array}{l}1^{\mathrm{b}} \\
1^{\mathrm{c}}\end{array}$ & NB & $\begin{array}{l}1 \text { retrospektive Kohor- } \\
\text { tenstudie }(n=2745)\end{array}$ & $\begin{array}{l}97 \% \\
95 \%\end{array}$ & $\begin{array}{l}88-99 \% \\
90-98 \%\end{array}$ & - & $\begin{array}{l}\text { Marolla et al. } \\
1998[52]\end{array}$ \\
\hline Singapur 2004 & 1 & NB & $\begin{array}{l}1 \text { retrospektive Kohor- } \\
\text { tenstudie }(n=184)\end{array}$ & $97 \%$ & - & - & $\begin{array}{l}\text { Ong et al. } 2007 \\
\text { [53] }\end{array}$ \\
\hline $\begin{array}{l}\text { Marshall-Inseln } \\
2003\end{array}$ & $\begin{array}{l}1 \\
2\end{array}$ & NB & $\begin{array}{l}1 \text { retrospektive Kohor- } \\
\text { tenstudie ( } 72 \text { Haushalt- } \\
\text { kontakte) }\end{array}$ & $\begin{array}{l}92 \% \\
95 \%\end{array}$ & $\begin{array}{l}67-98 \% \\
92-98 \%\end{array}$ & - & $\begin{array}{l}\text { Marin et al. } \\
2006[54]\end{array}$ \\
\hline \multicolumn{8}{|c|}{ Resultate eines systematischen Reviews zur Effektivität von Masernimpfstoffen (Uzicanin und Zimmerman 2011) [7] } \\
\hline Weltweit & 1 & $\geq 9$ Monate & $\begin{array}{l}106 \text { (Kohorten oder Fall- } \\
\text { Kontroll-Studien) }\end{array}$ & $91 \%$ & - & $79-95 \%$ & $\begin{array}{l}\text { Metaanalyse } \\
\text { [7] }\end{array}$ \\
\hline Weltweit & 2 & $\geq 9$ Monate & $\begin{array}{l}8 \text { (7 Kohortenstudien } \\
\text { und } 1 \text { Fall-Kontroll- } \\
\text { Studie) }\end{array}$ & $94 \%$ & & $88-98 \%$ & $\begin{array}{l}\text { Metaanalyse } \\
\text { [7] }\end{array}$ \\
\hline Europa & 1 & 9 bis 11 Monate & 3 Kohortenstudien & $96 \%$ & - & $88-98 \%$ & $\begin{array}{l}\text { Metaanalyse } \\
\text { [7] }\end{array}$ \\
\hline Europa & 1 & $\geq 12$ Monate & $\begin{array}{l}11 \text { (9 Kohorten- und } \\
2 \text { Fall-Kontroll-Studien) }\end{array}$ & $92 \%$ & - & $90-97 \%$ & $\begin{array}{l}\text { Metaanalyse } \\
\text { [7] }\end{array}$ \\
\hline $\begin{array}{l}\text { Deutschland } \\
2001 \text { bis } 2002\end{array}$ & 1 & NB & $\begin{array}{l}1 \text { retrospektive Kohor- } \\
\text { tenstudie ( } 124 \text { Haus- } \\
\text { haltkontakte) }\end{array}$ & $90 \%$ & $35-97 \%$ & - & $\begin{array}{l}\text { Arenz et al. } \\
2005 \text { [41] }\end{array}$ \\
\hline $\begin{array}{l}\text { Deutschland } \\
2001 \text { bis } 2002\end{array}$ & 1 & NB & $\begin{array}{l}1 \text { Erhebung mittels } \\
\text { Screeningmethode }\end{array}$ & $97 \%$ & $96-98 \%$ & - & $\begin{array}{l}\text { Arenz et al. } \\
2005 \text { [41] }\end{array}$ \\
\hline $\begin{array}{l}\text { Deutschland } \\
2006\end{array}$ & $\begin{array}{l}1 \\
2\end{array}$ & NB & $\begin{array}{l}1 \text { retrospektive Schul- } \\
\text { kohorte }(n=1014)\end{array}$ & $\begin{array}{l}98 \% \\
99 \%\end{array}$ & $\begin{array}{l}92-100 \% \\
97-100 \%\end{array}$ & - & $\begin{array}{l}\text { Wichmann et } \\
\text { al. } 2007 \text { [42] }\end{array}$ \\
\hline
\end{tabular}

nen verschiedene Aspekte herangezogen werden (vgl. im Folgenden Schöffski 2008 [38]). Liegt der Fokus ausschließlich auf den Kosten, werden die eingesparten Behandlungskosten ins Verhältnis zu den Innovationskosten (z. B. Kosten für eine innovative Impfung) gesetzt (vgl. Benefit-Cost-Ratio, BCR). Ein Wert über 1 repräsentiert demnach Kosteneinsparungen und spräche für die Verwendung der Innovation. Wenn neben den Kosten auch klinische Endpunkte, wie z. B. Anzahl der Krankheitsfälle, Anzahl der Hospitalisierungen bzw. Anzahl von Todesfällen, berechnet werden sollen, verwendet man die Kosten-EffektivitätsAnalyse. Hierbei werden neben den Kosten der innovativen und alternativen Behandlungsmethoden auch deren Effekte (z. B. auf die Anzahl der Krankheitsfälle oder Hospitalisierungen) berücksichtigt und in Relation gesetzt. Des Weiteren wird oft statt der klinischen Effekte die gesundheitsbezogene Lebensqualität berücksichtigt, um damit den subjektiven
Patientennutzen widerzuspiegeln. Dies erfolgt in der Regel mittels des qualitätsadjustierten Lebensjahrs (QALY); dabei spricht man von Kosten-Nutzwert-Analysen (• Tab. 1). Hieraus resultiert beispielsweise ein Eurobetrag pro vermiedenem Krankheitsfall oder pro gewonnenem QALY (incremental cost-effectiveness ratio, ICER). Manche Länder wenden einen Schwellenwert an. Liegt der ICER einer neuen Impfung im Vergleich zum Status quo darunter, wird diese Impfung vom Gesundheitssystem erstattet. In der Regel dienen diese Schwellenwerte eher als weiche Orientierungshilfen denn als hartes „Ja-oder-Nein“-Entscheidungskriterium. Das nationale Gesundheitssystem (NHS) in England und Wales hat beispielsweise einen Schwellenwert bzw. Korridor von 20.000-30.000 britischen Pfund Sterling pro QALY festgelegt, jedoch werden in Einzelfällen auch höhere Schwellenwerte akzeptiert $[39,40]$.

\section{Wirksamkeit der Masern- und Rötelnimpfung}

Im Mai 2011 wurde ein umfangreiches systematisches Review zur Wirksamkeit, Effektivität und Sicherheit der MMRKombinationsimpfstoffe von der Cochrane Collaboration durchgeführt [6]. Hierbei wurden mehrere RCTs zur Sicherheit der MMR-Impfstoffe identifiziert, aber keine RCTs, die die Wirksamkeit der seit den 1970er-Jahren auf dem Markt befindlichen MMR-Impfstoffe belegen. Es gibt jedoch mehrere epidemiologische Beobachtungsstudien, die eine hohe Effektivität der MMR-Impfstoffe zumindest bezüglich der Verhinderung von Masern belegen: Im Cochrane-Review wurden 3 Kohortenstudien zur MMR-Impfeffektivität bezüglich Verhinderung einer Masernerkrankung identifiziert, die mit nur einem moderaten Bias-Risiko behaftet waren [6]. Aufgrund der Heterogenität der 3 Kohortenstudien wurden diese nicht im Rahmen 
Tab. 3 Übersicht von Studien zur Wirksamkeit bzw. Effektivität der Rötelnimpfung in Bezug auf die Verhinderung klinischer Röteln während eines Ausbruchs. (Adaptiert nach Plotkin und Reef 2004 [4])

\begin{tabular}{|c|c|c|c|c|c|}
\hline Population, Ort & Studiendesign & $\begin{array}{l}\text { Exponierte } \\
\text { Individuen (n) }\end{array}$ & Impfstamm & $\begin{array}{l}\text { Impfeffektivität } \\
(95 \%-K I)\end{array}$ & $\begin{array}{l}\text { Autor und Jahr der } \\
\text { Publikation }\end{array}$ \\
\hline Schulkinder, Taiwan & $\begin{array}{l}\text { RCT mit klinischem } \\
\text { Endpunkt Röteln }\end{array}$ & $\begin{array}{l}3259 \text { geimpft, } \\
2735 \text { Placebo }\end{array}$ & HPV77 & $94 \%$ & $\begin{array}{l}\text { Grayston et al. } 1969 \\
\text { [55] }\end{array}$ \\
\hline Grundschule, Taiwan & $\begin{array}{l}\text { RCT mit klinischem } \\
\text { Endpunkt Röteln }\end{array}$ & $\begin{array}{l}198 \text { geimpft } \\
276 \text { Placebo }^{a}\end{array}$ & RA27/3 s.c. & $97 \%(77-100 \%)$ & Beasley et al. 1969 [43] \\
\hline $\begin{array}{l}\text { Kinderbetreuungsein- } \\
\text { richtung, USA }\end{array}$ & $\begin{array}{l}\text { Retrospektive } \\
\text { Kohortenstudie }\end{array}$ & 32 geimpft & Cendehill & $100 \%$ & Chang et al. 1970 [56] \\
\hline Jungenschule, Japan & $\begin{array}{l}\text { Prospektive } \\
\text { Kohortenstudie }\end{array}$ & 24 geimpft & RA27/3 & $100 \%$ & $\begin{array}{l}\text { Furukawa et al. } 1970 \\
\text { [57] }\end{array}$ \\
\hline Fördereinrichtung, USA & $\begin{array}{l}\text { Prospektive } \\
\text { Kohortenstudie }\end{array}$ & 22 geimpft & $\begin{array}{l}\text { HPV77 }(N=11) \text {, Cende- } \\
\text { hill }(n=4) \text {, Benoint C/D } \\
\text { level }(n=7)\end{array}$ & $100 \%$ & Davis et al. 1971 [58] \\
\hline Schulkinder, USA & $\begin{array}{l}\text { Retrospektive } \\
\text { Kohortenstudie }\end{array}$ & 362 geimpft & NB & $93 \%$ (84-97\%) & $\begin{array}{l}\text { Landrigan et al. } 1974 \\
\text { [59] }\end{array}$ \\
\hline Schulkinder, USA & $\begin{array}{l}\text { Fall-Kontroll-Studie } \\
\text { ( } 40 \text { Fälle, } 87 \text { Kontrol- } \\
\text { len) }\end{array}$ & $>600$ & NB & $90 \%(71-96 \%)$ & Greaves et al. 1983 [60] \\
\hline Universität, USA & $\begin{array}{l}\text { Fall-Kontroll-Studie } \\
\text { ( } 39 \text { Fälle, } 86 \text { Kontrol- } \\
\text { len) }\end{array}$ & $\begin{array}{l}\text { Unklar (ca. } 30.000 \text { Stu- } \\
\text { denten) }\end{array}$ & NB & $97 \%(82-100 \%)$ & $\begin{array}{l}\text { Strassburg et al. } 1985 \\
\text { [61] }\end{array}$ \\
\hline $\begin{array}{l}\text { Grundschule, Frank- } \\
\text { reich }\end{array}$ & $\begin{array}{l}\text { Retrospektive } \\
\text { Kohortenstudie }\end{array}$ & 119 & RA27/3 & $95 \%$ (85-99\%) & $\begin{array}{l}\text { De Valk und Revière } \\
1998 \text { [62] }\end{array}$ \\
\hline
\end{tabular}

einer Metaanalyse zusammengefasst (gepoolt), sondern getrennt beschrieben (- Tab. 2). Basierend auf diesen Kohortenstudien kamen die Autoren zu dem Schluss, dass eine MMR-Impfdosis eine Effektivität von mindestens 95 bzw. 98\% hat, Masern unter Vorschulkindern bzw. laborbestätigte Masern unter Schulkindern und Jugendlichen zu verhindern, und dass 1 bzw. 2 MMR-Impfdosen eine Effektivität von 92 bzw. 95\% haben, Sekundärfälle zu verhindern [6].

Ein systematisches Review zur Effektivität von Masernimpfstoffen (Monoimpfstoffe oder Kombinationsimpfstoffe) wurde 2011 publiziert [7]. In • Tab. 2 sind die gepoolten Effektschätzer für eine Impfdosis dargestellt, die in Beobachtungsstudien in Europa erhoben wurden. Diese liegen leicht, aber nicht statistisch signifikant, über dem gepoolten Effektschätzer, der auf Basis aller 106 identifizierten Kohorten- und Fall-Kontroll-Studien weltweit berechnet wurde. Für die Abschätzung der Effektivität von $2 \mathrm{Ma}$ sernimpfdosen wurden 8 Beobachtungsstudien weltweit identifiziert, die eine Effektivität von $94 \%$ ergaben. $\mathrm{Zu}$ den eingeschlossenen Studien gehörten auch 2 Untersuchungen aus Deutschland, die
2001 bis 2002 im Rahmen eines Ausbruchs in Coburg und 2006 im Rahmen eines Ausbruchs in Duisburg durchgeführt wurden $[41,42]$. Diese beiden Studien sind nochmals separat in $\bullet$ Tab. 2 aufgeführt.

Im Cochrane-Review wurden keine Studien zu MMR-Impfeffektivität bezüglich Verhinderung von Röteln identifiziert. Es liegen jedoch diverse Beobachtungsstudien vor, die im Rahmen von Ausbrüchen insbesondere an Schulen durchgeführt wurden und die gute Effektivität von Röteln-Impfstoffen belegen (• Tab.3). In einem für das Rötelnpositionspapier der WHO durchgeführten systematischen Review wurde ein RCT identifiziert, der die Wirksamkeit eines RA27/3-Rötelnimpfstoffs erhob [4]. RA27/3 ist der weltweit am häufigsten für Rötelnimpfstoffe benutzte Impfvirusstamm, der ausschließlich bei den aktuell in Deutschland verfügbaren Impfstoffen zur Anwendung kommt. In diese doppelblind, randomisierte Studie waren 198 RA27/3-geimpfte und 276 placebogeimpfte Kinder in 4 taiwanesischen Grundschulen während eines Ausbruchs eingeschlossen, und es konnte eine Wirksamkeit von 97\% nachgewiesen werden [43].

\section{Einfluss der Masern- und \\ Rötelnimpfung auf \\ Bevölkerungsebene und in Ausbrüchen}

Neben Studien zur Wirksamkeit und Effektivität stehen Daten aus der Krankheitssurveillance zur Verfügung, die den Impact (d. h. Gesamteffekt auf Bevölkerungsebene) der Impfstoffe eindeutig belegen. Mithilfe eines Modells und basierend auf länderspezifischen Impfquoten und Fallmeldungen wurde von der WHO geschätzt, dass zwischen 2000 und 2010 durch die Impfung eine Reduktion der Maserntodesfälle weltweit um $74 \%$ erreicht wurde [3]. Während im Jahr 2000 weltweit geschätzte 535.000 Masern-assoziierte Todesfälle auftraten, waren es 2010 nur noch 139.300 Todesfälle [3].

Ein deutlicher Rückgang sowohl der Masern- als auch Rötelnfälle konnte insbesondere in den Ländern Nord- und Südamerikas verzeichnet werden. Durch Implementierung entsprechender Strategien, die auf das Erreichen von hohen Impfquoten im Routineimpfprogramm und auf die Durchführung von Catchup-Impfkampagnen unter Kindern und Jugendlichen abzielten, konnten bei- 


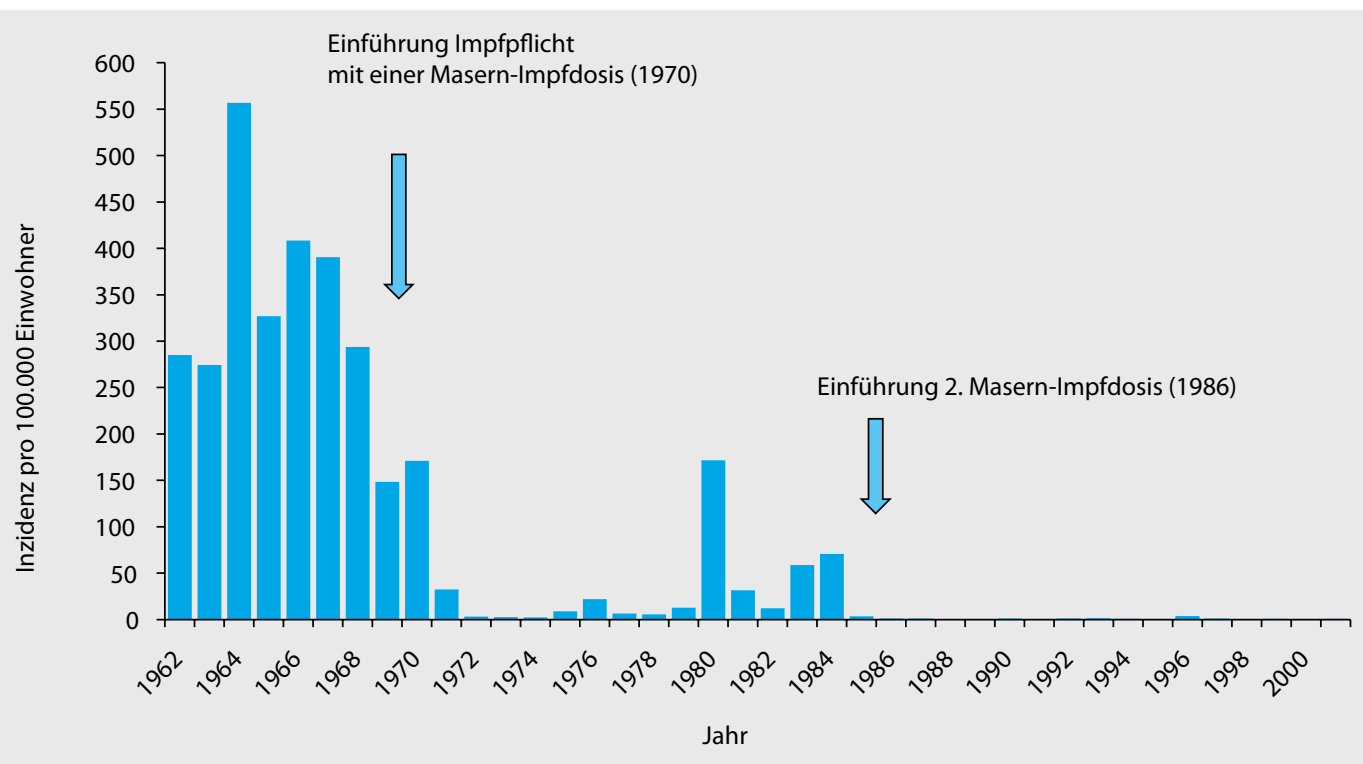

Abb. $1 \varangle$ Populationseffekt (,Impact") der Masernimpfung: gemeldete Masernfälle in der DDR bzw. in den östlichen Bundesländern (1962 bis 2001)

de Erkrankungen auf dem amerikanischen Kontinent eliminiert werden [2]. Der letzte endemische Masernfall wurde 2002 und der letzte endemische Rötelnfall wurde 2009 auf dem Kontinent berichtet [2].

Auch in Finnland konnten durch die Implementierung eines 2-Dosen-MMRImpfprogramms im Jahr 1982 beide Erkrankungen vollständig eliminiert werden [44]. Während die Inzidenz von Masern und Röteln 1982 noch bei 105 bzw. 64 pro 100.000 Einwohnern lag, konnte diese innerhalb von 13 Jahren auf unter 0,1 pro 100.000 Einwohner gesenkt werden. Neben einem qualitativ hochwertigen Routineimpfprogramm wurden in Finnland in den 1980er-Jahren auch Catchup-Impfprogramme für Schulkinder, Studenten und Gesundheitspersonal durchgeführt, von 1986 bis 2000 auch unter Rekruten [44].

In Deutschland waren die Röteln bis 2013 nicht meldepflichtig. Daher liegen keine validen Inzidenzdaten vor, aus denen der Trend bei der Erkrankung seit Einführung der Rötelnimpfung abzuleiten wäre. Daten zum seit 2001 nicht namentlich an das Robert Koch-Institut zu meldenden konnatalen Rötelnsyndrom sind in einem anderen Beitrag in diesem Schwerpunktheft dargestellt. Zur Masernerkrankung liegen Meldedaten aus der ehemaligen DDR seit 1962 vor; sie sind in $\bullet$ Abb. 1 aufgetragen [45]. Die Grafik belegt den Effekt, den die 1970 ein- geführte Impfpflicht mit einer Masernimpfdosis (inklusive Massenimpfaktion aller Vorschul- und Schulkinder) hatte, gefolgt von einer Zweitimpfung für alle 2- bis 16-Jährigen, die vor dem vollendeten 1. Lebensjahr erstgeimpft wurden (1983) und einer Pflicht-Zweitimpfung für alle Kinder (1986) [46]. In der BRD stiegen mit einer allgemeinen Masernimpfempfehlung 1974 die Impfquoten hingegen deutlich langsamer an, und breit angelegte Catch-up-Impfaktivitäten zur Schließung von Immunitätslücken in höheren Altersgruppen blieben aus. Als Konsequenz erfüllen die östlichen Bundesländer bereits heute mehrere Kriterien für die Maserneliminierung, während in den westlichen Bundesländern die jährliche Maserninzidenz seit 2003 im Durchschnitt fast 10-fach höher liegt [47].

$\mathrm{Zu}$ den typischen epidemiologischen Effekten nach breiter Einführung der Masern/Röteln-Impfung in eine Bevölkerung gehören eine Reduktion der Größe von Ausbrüchen und eine Verlängerung der interepidemischen Intervalle zwischen den einzelnen Ausbrüchen [1]. Bei kontinuierlich steigenden Impfquoten in der jungen Bevölkerung kommt es im Verlauf sowohl zu einer Inzidenzreduktion in den geimpften Kohorten als auch zu einer generellen Reduktion an Masernfällen in der Gesamtbevölkerung. Im Rahmen von Ausbrüchen kommt es in der Folge typischerweise - wie auch aktuell in Deutschland zu beobachten ist - zu einem Anstieg des Anteils an in der Kindheit nicht geimpften Jugendlichen und jungen Erwachsenen unter den Erkrankungsfällen $[1,47]$. Daher sind Catch-upImpfungen von Empfänglichen insbesondere unter älteren Kindern und Jugendlichen ein wesentlicher Bestandteil der Strategie zur Eliminierung der Masern und Röteln [5].

Aufgrund der hohen Basisreproduktionszahl von Masern erscheinen Impfaktivitäten zur Eindämmung institutioneller Ausbrüche oft nicht sinnvoll, da von der Meldung eines ersten Falls bis hin zur Organisation einer Impfintervention und dem Eintreten des Schutzes (in der Regel ca. 2 Wochen nach Impfung) viel Zeit vergeht. Dabei wird jedoch vergessen, dass eine vorbestehende hohe Impfquote in der Institution zu einer deutlichen Verlangsamung der Transmission (d. h. niedrigere effektive Reproduktionszahl, $\mathrm{R}_{\mathrm{eff}}$ ) führt. Ein mathematisches Modell, das auf Daten eines Duisburger Schulausbruchs basiert, zeigt, dass bei einer vorbestehenden Impfquote von $91-94 \%$ eine Impfintervention selbst innerhalb eines Zeitraums von 40 bis 50 Tagen immer noch mit einer Wahrscheinlichkeit von $95 \% \mathrm{zu}$ einer Reduzierung der Ausbruchsgröße führt [48]. Je niedriger die vorbestehende Immunität in einer Institution ist, desto explosionsartiger entwickeln sich $\mathrm{Ma}$ sernausbrüche und desto früher muss eine Impfintervention eingeleitet werden, damit diese noch effektiv ist [48]. 


\begin{tabular}{|c|c|c|c|c|c|c|}
\hline Ursprungsland & Preisjahr & Impfung & $\begin{array}{l}\text { Studiendesign } \\
\text { (Analyseansatz) }\end{array}$ & $\begin{array}{l}\text { Zeithorizont } \\
\text { (Jahre) }\end{array}$ & Hauptergebnis & $\begin{array}{l}\text { Autor und Jahr } \\
\text { der Publikation }\end{array}$ \\
\hline USA & 1997 & $\mathrm{MMR}^{\mathrm{a}}$ & Kosten-Nutzen & 13 & $\begin{array}{l}45 \text { Mio. \$ jährliche Einspa- } \\
\text { rung durch Eliminierung }\end{array}$ & $\begin{array}{l}\text { Miller et al. } 1998 \\
{[10]}\end{array}$ \\
\hline Kanada & 1995 & $\mathrm{MMR}^{\mathrm{a}}$ & Kosten-Nutzen & 20 & BCR: 2,61-4,31 & $\begin{array}{l}\text { Pelletier et al. } 1998 \\
\text { [11] }\end{array}$ \\
\hline $\begin{array}{l}\text { Hypothetisches } \\
\text { Industrieland }\end{array}$ & 1999 & Masern $^{\mathrm{a}}$ & $\begin{array}{l}\text { Kosten-Nutzen und } \\
\text { Kosteneffektivität }\end{array}$ & 50 & $\begin{array}{l}\mathrm{BCR}: 0 ; 49-12,01 \\
20-536 \text { EUR pro vermiede- } \\
\text { nem Fall }\end{array}$ & $\begin{array}{l}\text { Beutels et al. } 2003 \\
\text { [8] }\end{array}$ \\
\hline Schweiz & 1978 & Masern & Kosten-Nutzen & 1 & BCR: 1,97 & Just 1978 [9] \\
\hline Japan & NB & Masern & Kosten-Nutzen & 4 & $\mathrm{BCR}^{\mathrm{b}}: 2,21-4,97$ & $\begin{array}{l}\text { Takahashi et al } \\
2011 \text { [12] }\end{array}$ \\
\hline USA & 1983 & MMR & Kosten-Nutzen & 1 & BCR: 14 & $\begin{array}{l}\text { White et al. } 1985 \\
\text { [13] }\end{array}$ \\
\hline USA & 2001 & $\mathrm{MMR}^{\mathrm{a}}$ & Kosten-Nutzen & 40 & $\mathrm{BCR}^{\mathrm{b}}: 10,5-54,2$ & $\begin{array}{l}\text { Zhou et al. } 2004 \\
\text { [14] }\end{array}$ \\
\hline Österreich & NB & MM & Kosten-Nutzen & 12 & $\mathrm{BCR}^{\mathrm{b}}: 2,65-4,48$ & $\begin{array}{l}\text { Wiedermann et al. } \\
1979 \text { [15] }\end{array}$ \\
\hline Dänemark & 1984 & MMR & Kosten-Nutzen & 20 & $\begin{array}{l}\text { Nettoeinsparung: } \\
\text { 135,4-140,8 Mio. Dänische } \\
\text { Kronen nach } 20 \text { Jahren }\end{array}$ & $\begin{array}{l}\text { Bjerregaard } 1991 \\
\text { [16] }\end{array}$ \\
\hline USA & 1975 & Röteln & Kosten-Nutzen & Lebenslang & $\mathrm{BCR}: 8-27$ & $\begin{array}{l}\text { Schoenebaum et al } \\
1976 \text { [19] }\end{array}$ \\
\hline Frankreich & NB & Masern & Kosteneffektivität & 15 & $\begin{array}{l}15.500 \text { Franc pro vermie- } \\
\text { denem Todesfall }\end{array}$ & $\begin{array}{l}\text { Chapalain } 1978 \\
\text { [17] }\end{array}$ \\
\hline Finnland & 1973 & Röteln & Kosten-Nutzen & 30 & $\mathrm{BCR}=3,3-12,9$ & Elo 1979 [18] \\
\hline Norwegen & 1978 & Röteln & Kosten-Nutzen & Lebenslang & $\mathrm{BCR}^{\mathrm{b}}: 5-20$ & $\begin{array}{l}\text { Stray-Pedersen } \\
1982[20]\end{array}$ \\
\hline
\end{tabular}

\section{Gesundheitsökonomie der Masern- und Rötelnimpfung}

Die ökonomische Belastung der weltweiten Gesundheitssysteme durch Masern sind in Industrieländern mitunter hoch [49]. Bei den Behandlungskosten pro Masernfall rangiert Deutschland im Vergleich zu 10 anderen europäischen bzw. nordamerikanischen Ländern mit 236 EUR (Jahr 2001) pro Masernfall im Mittelfeld [50]. Betrachtet man die Gesamtausgaben für die Masernbehandlung, lag Deutschland Anfang dieses Jahrtausends in Europa nach Italien und Frankreich mit jährlich über 20 Mio. EUR auf dem dritten Platz [49].

Eine systematische Literaturrecherche im Rahmen eines Health Technology Assessments (HTA) aus dem Jahr 2007 ergab, dass es für Deutschland keine gesundheitsökonomische Evaluation der Masern- noch der Rötelnimpfung gab [50]. Bis heute wird in der Literatur hier- zu nichts beschrieben. Aufgrund unterschiedlicher epidemiologischer, gesundheitssystemischer und finanzieller Rahmenbedingungen, aber auch aufgrund unterschiedlicher Modellansätze sind Ergebnisse gesundheitsökonomischer Evaluationen aus anderen Ländern ohne Anpassungen nur limitiert auf Deutschland übertragbar [51].

Die Ergebnisse des in der Einleitung beschriebenen eigenen systematischen Reviews internationaler Studien können gleichwohl als Ausgangspunkt dienen, wenn man die Kosteneffektivität der Masern- und Rötelnimpfung in Deutschland diskutiert. Eine Übersicht der im Review eingeschlossenen Studien gibt $\bullet$ Tab. 4.

Eine Analyse, die das gesundheitsökonomische Potenzial einer Maserneliminierung durch Impfung in den USA modellierte, errechnete jährliche Kosteneinsparungen von 45 Mio. US Dollar [10]. In Kanada wurde im Zuge der Änderung des Masernimpfprogramms von 1 auf 2 Dosen berechnet, dass das 2-DosenImpfschema die zusätzlich entstandenen Kosten durch höhere zusätzliche Nutzen kompensierte und zu Kosteneinsparungen beitrug [11]. Eine andere Studie nahm ein fiktives europäisches Land als Grundlage und untersuchte die Kosteneffektivität von 10 verschiedenen Impfstrategien zur Steigerung der Masernimpfquoten. Es wurde festgestellt, dass in einem Masernimpfprogramm bei einer relativ geringen 1-Dosis-Impfquote $(<70 \%)$ als Ausgangslage die Steigerung der Impfquote auf 90-95\% zu Kosteneinsparungen führte. Lägen die Impfquoten anfänglich jedoch bereits bei über $90 \%$, hinge es vom jeweiligen Gesundheitssystem $\mathrm{ab}$, ob eine derartige Maßnahme noch als kosteneffektiv angesehen werden würde oder nicht [8]. Für die Schweiz wurde bereits 1978 errechnet, dass sich eine generelle Masernimpfung im zweiten Lebensjahr volkswirtschaftlich „rentiert“ [9]. In Japan, wo vor Implementierung eines na- 
tionalen Masernimpfprogramms im Jahre 2006 die Masernimpfquote um die 80\% lag, identifizierte ein Modell ebenfalls ein Kosteneinsparungspotenzial durch das Impfen [12]. Anfang der 1980er-Jahre wurde in den USA das 1-Dosen-MasernMumps-Röteln (MMR)-Impfprogramm auf seine Kosteneffektivität untersucht. Infolge der deutlichen Fallreduktion und der damit verbundenen Abnahme der Behandlungskosten wurden die Ausgaben für das Impfprogramm mehr als ausgeglichen. Zusätzlich wurde errechnet, dass die Verwendung der kombinierten MMR-Impfung im Vergleich zu der von Einzelimpfstoffen 60 Mio. US Dollar einsparte [13]. Eine Analyse des 2-DosenMMR-Impfprogramms in den USA attestierte beinahe 30 Jahre später dessen kosteneinsparende Wirkung. Darüber hinaus wurde gerade die Kosteneffektivität der zweiten im Vergleich zur ersten Dose herausgestellt [14]. Eine Studie aus den späten 1970er-Jahren errechnete für Österreich, dass ein jährliches Impfen von 100.000 einjährigen Kindern gegen Mumps und Masern nach 12 Jahren - abhängig von der Analyse - etwa zwischen 60 und 530 Mio. österreichische Schilling einsparen könnte [15]. Auch in Dänemark war der errechnete Nutzen der 2-DosenMMR-Impfung über 20 Jahre gesehen höher als die errechneten Kosten [16].

Eine gesundheitsökonomische Analyse der Rötelnimpfung in den USA ergab eine Kosten-Nutzen-Relation zugunsten der Impfung unabhängig von der Impfquote (100 vs. $80 \%)$. Das Impfalter der Kinder und die Verwendung der MMR-Kombinationsimpfung hatten hingegen Auswirkung auf das Kosten-Nutzen-Verhältnis bzw. BCR [19]. Eine Modellierung in Frankreich ergab, dass die Rötelnimmunisierung von 13-jährigen Mädchen und Frauen, die in Einrichtungen für Kinder arbeiten, über einen Zeitraum von 15 Jahren einen ICER von 15.500 Franc pro vermiedenem Todesfall ergab [17]. In Finnland hätte die Impfung von 13-jährigen Mädchen und Frauen in der postpartalen Phase über einen Zeitraum von 25 bis 30 Jahren 90 Mio. Finnische Mark eingespart [18]. Eine norwegische Modellierung identifizierte ebenfalls das Rötelnimpfen von 13-jährigen Mädchen als kosteneffektive Strategie, wobei das Impfen ausschließlich von suszeptiblen 13-jährigen Mädchen noch effizienter wäre. Darüber hinaus erschien die Verwendung von Kombinationsimpfstoffen ebenfalls wirtschaftlicher [20]. Die WHO rät auch aufgrund dieser gesundheitsökonomischen Evidenz, die Impfung gegen Röteln in Ländern mit einer bereits bestehenden 2-Dosen-Masernimpfung in das Impfprogramm mit aufzunehmen [4].

Aufbauend auf die bereits bestehenden Empfehlungen hat die WHO einen Strategieplan "Global Measles and Rubella 2012-2020“ zur Eliminierung dieser beiden Krankheiten entwickelt. In einem eigenen Abschnitt wird hierin auf die günstigen Kosten-Nutzen-Relationen bzw. auf das Kosteneinsparungspotenzial der MMR-Impfung in verschiedenen Ländern eingegangen. Ferner wird aufgeführt, dass entwickelte Ländern und damit auch Deutschland immer noch hohe Kosten für die Bewältigung von Ausbrüchen dieser Erkrankungen aufwenden müssen. Eine verstärkte Anstrengung zur Eliminierung erscheint damit auch aus ökonomischer Sicht geboten [5].

\section{Diskussion und Fazit}

Auch wenn die Wirksamkeit der Masernund Rötelnimpfung nicht bzw. nur eingeschränkt durch RCTs nachgewiesen wurde, so belegen dennoch zahlreiche epidemiologische Beobachtungsstudien die gute Effektivität der Impfung. In einer formalen Bewertung der Qualität der Evidenz würden zwar die Beobachtungsstudien aufgrund ihres Studiendesigns per se niedriger eingestuft werden; die über diverse Studiensettings und Studiendesigns konsistent großen Effekte und eine Dosis-Wirkung-Beziehung (d. h. stärkerer Gesamteffekt eines Impfprogramms bei höheren Impfquoten, wie z. B. im Osten Deutschlands im Vergleich zum Westen) führen jedoch dazu, dass die Evidenz bezüglich der Effektivität als sehr hoch eingestuft werden kann [4]. Die Eliminierung beider Erkrankungen auf dem amerikanischen Kontinent und in Finnland kann zudem als Beweis dafür angesehen werden, dass eine Eliminierung im Rahmen eines 2-Dosen-Impfprogramms und von zusätzlichen Impfaktivitäten möglich ist.

Mithilfe mathematischer Modellierungen konnte ein besseres Verständnis für die komplexe Situation bei Masernausbrüchen in Institutionen mit vorbestehender Populationsimmunität entwickelt werden. Die Modellierung legte nahe, dass bei vorhandenen Impfquoten von $>90 \%$ ausreichend Zeit ist, eine Impfintervention einzuleiten, die die finale Ausbruchsgröße mit hoher Wahrscheinlichkeit reduziert. Eine gesundheitsökonomische Evaluation der Masern- und Rötelnimpfung liegt für Deutschland nicht vor. Jedoch lassen sich die Erkenntnisse zum kosteneffektiven bzw. kosteneinsparenden Potenzial dieser Maßnahmen aus internationalen Studien mit gewissen Einschränkungen auch für Deutschland ableiten, da die betrachteten Länder gewisse Ähnlichkeiten mit dem deutschen Gesundheitssystem aufweisen.

Die Beispiele aus Südamerika und Finnland zeigen, dass zur Elimination der Masern hohe Impfquoten bzw. eine hohe Bevölkerungsimmunität in allen Altersgruppen vorhanden sein müssen. Immunitätslücken können am effektivsten durch Catch-up-Impfkampagnen geschlossen werden. Auch in Deutschland sind Catchup-Impfungen von Kindern, Jugendlichen und jungen Erwachsenen speziell in den westlichen Bundesländern erforderlich. Ohne diese zusätzlichen Impfmaßnahmen kann und wird das Ziel der Elimination bis 2015 nicht erreicht werden.

\section{Korrespondenzadresse}

\section{PD Dr. O. Wichmann}

Fachgebiet Impfprävention,

Abteilung für Infektionsepidemiologie,

Robert Koch-Institut

Seestr. 10, 13353 Berlin

Wichmann0@rki.de

Interessenkonflikt. O. Wichmann und B. Ultsch geben an, dass kein Interessenkonflikt besteht.

Dieser Beitrag beinhaltet keine Studien an Menschen oder Tieren.

\section{Literatur}

1. Plotkin S, Orenstein W, Offit P (2012) Vaccines. Elsevier, Philadelphia

2. Andrus JK, Quadros CA de, Solorzano CC et al (2011) Measles and rubella eradication in the Americas. Vaccine 29(Suppl 4):D91-D96

3. Simons E, Ferrari M, Fricks J et al (2012) Assessment of the 2010 global measles mortality reduction goal: results from a model of surveillance data. Lancet 379:2173-2178 
4. World-Health-Organization (2011) Rubella vaccines: WHO position paper. Releve epidemiologique hebdomadaire/Section d'hygiene du Secretariat de la Societe des Nations = Weekly epidemiological record/Health Section of the Secretariat of the League of Nations 86:301-316

5. World Health Organization (2012) Global measles and rubella strategic plan: 2012-2020. World Health Organization, Genf

6. Demicheli V, Rivetti A, Debalini MG, Di Pietrantonj C (2012) Vaccines for measles, mumps and rubella in children. Cochrane Database Syst Rev 2:CD004407

7. Uzicanin A, Zimmerman L (2011) Field effectiveness of live attenuated measles-containing vaccines: a review of published literature. J Infect Dis 204(Suppl 1):S133-S148

8. Beutels P, Gay NJ (2003) Economic evaluation of options for measles vaccination strategy in a hypothetical Western European country. Epidemiol Infect 130:273-283

9. Just M (1978) Rentiert die Masern- und/oder Mumps-Impfung für schweizerische VErhältnisse? Schweiz Med Wochenschr 108

10. Miller MA, Redd S, Hadler S, Hinman A (1998) A model to estimate the potential economic benefits of measles eradication for the United States. Vaccine 16:1917-1922

11. Pelletier L, Chung P, Duclos P et al (1998) A benefitcost analysis of two-dose measles immunization in Canada. Vaccine 16:989-996

12. Takahashi K, Ohkusa Y, Kim J-Y (2011) The economic disease burden of measles in Japan and a benefit cost analysis of vaccination, a retrospective study. BMC Health Serv Res 11:254

13. White CC, Koplan JP, Orenstein WA (1985) Benefits, risks and costs of immunization for measles, mumps and rubella. Am J Public Health 75:739-744

14. Zhou F, Reef S, Massoudi M et al (2004) An economic analysis of the current universal 2-dose measles-mumps-rubella vaccination program in the United States. J Infect Dis 189(Suppl 1):S131-S145

15. Wiedermann G, Ambrosch F (1979) Costs and benefits of measles and mumps immunization in Austria. Bull World Health Organ 57:625-629

16. Bjerregaard $\mathrm{P}$ (1991) Economic analysis of immunization programmes. Scand J Soc Med Suppl 46:115-119

17. Chapalain MT (1978) Perinatality: French cost-benefit studies and decisions on handicap and prevention. Ciba Found Symp 193-206

18. Elo $O$ (1979) Cost-benefit studies of vaccinations in Finland. Developments in biological standardization 43:419-428

19. Schoenbaum SC, Hyde JNJBL, Crampton K (1976) Benefit-cost analysis of rubella vaccination policy. N Engl J Med 294:306-310

20. Stray-Pedersen B (1982) Economic evaluation of different vaccination programmes to prevent congenital rubella. NIPH Ann 5:69-83

21. Halloran ME, Struchiner CJ, Longini IM Jr (1997) Study designs for evaluating different efficacy and effectiveness aspects of vaccines. Am J Epidemiol 146:789-803

22. Chen RT, Orenstein WA (1996) Epidemiologic methods in immunization programs. Epidemiol Rev 18:99-117

23. Orenstein WA, Bernier RH, Dondero TJ et al (1985) Field evaluation of vaccine efficacy. Bull World Health Organ 63:1055-1068

24. Kunz R, Ollenschläger G, Raspe HH et al (2007) Lehrbuch für Evidenzbasierte Medizin in Klinik und Praxis. Deutscher Ärzte-Verlag, Köln
25. Farrington CP (1993) Estimation of vaccine effectiveness using the screening method. Int J Epidemiol 22:742-746

26. Weinberg GA, Szilagyi PG (2010) Vaccine epidemiology: efficacy, effectiveness, and the translational research roadmap. J Infect Dis 201:1607-1610

27. Salisbury DM, Beverley PCL, Miller E (2002) Vaccine programmes and policies. Br Med Bull 62:201-211

28. Briggs A, Claxton K, Sculpher M (2008) Decision modelling for health economic evaluation. Oxford University Press, Oxford

29. Siebert U, Mühlberger N, Schöffski O (2008) Evidenzsynthese: meta-Analysen und Entscheidungsanalysen. In: Schöffski O, von der Schulenburg JM (Hrsg) Gesundheitsökonomische Evaluation. Springer, Berlin Heidelberg New York Tokyo, S 261-310

30. Beutels P, Van Doorslaer E, Van Damme P, Hall J (2003) Methodological issues and new developments in the economic evaluation of vaccines. Expert Rev Vaccines 2:649-660

31. Pitzer VE, Viboud C, Lopman BA et al (2011) Influence of birth rates and transmission rates on the global seasonality of rotavirus incidence. J R Soc Interface 8:1584-1593

32. Sugerman DE, Barskey AE, Delea MG et al (2010) Measles outbreak in a highly vaccinated population, San Diego, 2008: role of the intentionally undervaccinated. Pediatrics 125:747-755

33. De Serres G, Gay NJ, Farrington CP (2000) Epidemiology of transmissible diseases after elimination. Am JEpidemiol 151:1039-1048 (discussion 1049-1052)

34. Icks A, Chernyak N, Bestehorn Ket al (2010) Methoden der gesundheitsökonomischen Evaluation in der Versorgungsforschung. Gesundheitswesen

35. Garrison LP, Bauch CT, Bresnahan BW et al (2011) Using cost-effectiveness analysis to support research and development portfolio prioritization for product innovations in measles vaccination. $\mathrm{J} \mathrm{In-}$ fect Dis 204:S124-S132

36. IQWiG (2009) Cost estimation - working paper, Version 1.0. Gesundheitswesen IfQuWi (Hrsg) Institut für Qualität und Wirtschaftlichkeit im Gesundheitswesen Cologne

37. Drummond M, Chevat C, Lothgren M (2007) Do we fully understand the economic value of vaccines? Vaccine 25:5945-5957

38. Schöffski O (2008) Grundformen gesundheitsökonomischer Evaluationen. In: Schöffski O, von der Schulenburg JM (Hrsg) Gesundheitsökonomische Evaluation. Springer, Berlin Heidelberg New York Tokyo, S 65-92

39. Raftery JP (2008) Paying for costly pharmaceuticals: regulation of new drugs in Australia, England and New Zealand. Med J Aust 188:26-28

40. NICE (2008) National Institute for Health and Clinical Excellence: guide to the methods of technology appraisal. In: System NH (Hrsg)

41. Arenz S, Schmitt HJ, Tischer A, Kries R von (2005) Effectiveness of measles vaccination after household exposure during a measles outbreak: a household contact study in Coburg, Bavaria. Pediatr Infect Dis J 24:697-699

42. Wichmann O, Hellenbrand W, Sagebiel D et al (2007) Large measles outbreak at a German public school, 2006. Pediatr Infect Dis J 26:782-786

43. Beasley RP, Detels R, Kim KS et al (1969) Prevention of rubella during an epidemic on Taiwan. HPV77 and RA 27-3 rubella vaccines administered subcutaneously and intranasally HPV-77 vaccine mixed with mumps and-or measles vaccines. Am J Dis Child 118:301-306

44. Davidkin I, Kontio M, Paunio M, Peltola H (2010) MMR vaccination and disease elimination: the Finnish experience. Expert Rev Vaccines 9:1045-1053
45. Pöhn HP, Rasch G (1994) Statistik meldepflichtiger übertragbarer Krankheiten: Vom Beginn der Aufzeichnungen bis heute (Stand 31. Dezember 1989). MMV Medizin, München

46. Klein S, Schoneberg I, Krause G (2012) The historical development of immunization in Germany. From compulsory smallpox vaccination to a National Action Plan on Immunization. Bundesgesundheitsbl Gesundheitsforsch Gesundheitsschutz 55:15121523

47. Siedler A, Mankertz A, Feil F et al (2011) Closer to the goal: efforts in measles elimination in Germany 2010. J Infect Dis 204(Suppl 1):S373-S380

48. Bonacic Marinovic AA, Swaan C, Wichmann O et al (2012) Effectiveness and timing of vaccination during school measles outbreak. Emerg Infect Dis 18:1405-1413

49. Carabin H, Edmunds WJ, Gyldmark M et al (2003) The cost of measles in industrialised countries. Vaccine 21:4167-4177

50. Rosian-Schikuta I, Fröschl B, Habl C, Stürzliner H (2007) Die Masern-Mumps-Röteln-Impfung aus gesundheitspolitischer und ökonomischer Sicht (2007). In: (DIMDI) DIfMDul (Hrsg) Health technology assessment, Bd 62

51. Welte R, Feenstra T, Jager H, Leidl R (2004) A decision chart for assessing and improving the transferability of economic evaluation results between countries. Pharmacoeconomics 22:857-876

52. Marolla F, Baviera G, Cacciapuoti et al (1998) A field study on vaccine efficacy against mumps of three MMR vaccines [Efficacia verso la parotite di tre diversi vaccini a tripla componente: studio sul campo]. Riv Ital Pediatr 24:466-472

53. Ong G, Rasidah N, Wan S, Cutter J (2007) Outbreak of measles in primary school students with high first dose MMR vaccination coverage. Singapore Med J 48:656-661

54. Marin M, Nguyen HQ, Langidrik JR et al (2006) Measles transmission and vaccine effectiveness during a large outbreak on a densely populated island: implications for vaccination policy. Clin Infect Dis 42:315-319

55. Grayston JT, Detels R, Chen KP et al (1969) Field trial of live attenuated rubella virus vaccine during an epidemic on Taiwan. Preliminary report of efficacy of three HPV-77 strain vaccines in the prevention of clinical rubella. JAMA 207:1107-1110

56. Chang TW, Des Rosiers S, Weinstein L (1970) Clinical and serologic studies of an outbreak of rubella in a vaccinated population. N Engl J Med 283:246-248

57. Furukawa T, Miyata T, Kondo K et al (1970) Rubella vaccination during an epidemic. JAMA 213:987-990

58. Davis WJ, Larson HE, Simsarian JP et al (1971) A study of rubella immunity and resistance to infection. JAMA 215:600-608

59. Landrigan PJ, Stoffels MA, Anderson E, Witte JJ (1974) Epidemic rubella in adolescent boys. Clinical features and results of vaccination. JAMA 227:1283-1287

60. Greaves WL, Orenstein WA, Hinman AR, Nersesian WS (1983) Clinical efficacy of rubella vaccine. Pediatr Infect Dis 2:284-286

61. Strassburg MA, Greenland S, Stephenson TG et al (1985) Clinical effectiveness of rubella vaccine in a college population. Vaccine 3:109-112

62. Valk H de, Rebière I (1998) Epidémie de rubéole: Evaluation de l'effi cacité vaccinale sur le terrain, $\mathrm{Ar}$ dèche, janvier mars 1997. Réseau National de santé Publique, Saint Maurice, France, 1-52 